\title{
Tratamiento endoscópico de la hiperplasia benigna de próstata bajo anestesia local-sedación en pacientes de alto riesgo
}

\author{
P. Navalón Verdejo*,**,***, F. Sánchez Ballester**, F. Ordoño Domínguez**, Y. Pallás Costa*, \\ J. Juan Escudero**, J.A. Cánovas Ivorra* \\ *Servicio de Urología. Hospital Casa de Salud. Valencia. \\ **Servicio de Urología. Consorcio Hospital General Universitario. Valencia. \\ ***Departamento de Cirugía. Facultad de Medicina. Universidad de Valencia.
}

Actas Urol Esp 2006; 30 (1): 46-52

\section{RESUMEN}

TRATAMIENTO ENDOSCÓPICO DE LA HIPERPLASIA BENIGNA DE PRÓSTATA BAJO ANESTESIA LOCAL-SEDACIÓN EN PACIENTES DE ALTO RIESGO

Introducción y objetivo: El envejecimiento de la población comporta un incremento del número de varones afectos de hiperplasia benigna de próstata (HBP) con múltiple patología asociada que limita su tratamiento quirúrgico. Con este estudio pretendemos poner de manifiesto la eficacia y seguridad del empleo de anestesia local-sedación para el tratamiento endoscópico de la HBP en pacientes de alto riesgo quirúrgico-anestésico.

Método: Durante los últimos 4 años hemos intervenido a 38 pacientes con edades comprendidas entre 78 y 86 años (media $81,4 \pm 3,2$ ) con síndrome de prostatismo obstructivo severo y riesgo quirúrgico-anestésico elevado. En 15 pacientes se practicó incisión transuretral de próstata (ITUP) y en los 23 restantes resección transuretral (RTUP). El bloqueo anestésico se realizó mediante infiltración periprostática transrectal digito-dirigida con 10-15 ml de lidocaína al 2\%. Durante el procedimiento se administró una perfusión intravenosa de propofol y remifentanilo. Se evalúan los resultados obtenidos así como el grado de satisfacción de los pacientes mediante la elaboración de un cuestionario.

Resultados: La duración media de la intervención fue de $12 \pm 5$ minutos (rango 8 - 25), no habiéndose constatado complicaciones derivadas de la técnica anestésica. Refirieron una molestia leve 4 pacientes (11\%) que se resolvió aumentando la velocidad de la perfusión sedante. El grado de satisfacción con el tratamiento recibido ha sido del 95\%.

Conclusiones: Consideramos que tanto la ITUP como RTUP realizadas bajo anestesia local-sedación son métodos seguros, eficaces y bien tolerados, que están fundamentalmente indicados en pacientes añosos y cuando el riesgo quirúrgico-anestésico es elevado.

Palabras clave: Hiperplasia prostática benigna. Anestesia local. Resección transuretral de próstata.

\section{ABSTRACT \\ ENDOSCOPIC TREATMENT OF THE BENIGN PROSTATIC HYPERPLASIA WITH LOCAL ANESTHESIA AND SEDATION IN PATIENTS WITH HIGH SURGICAL OR ANAESTHESICAL RISK}

Introduction and objetive: The aging of the population causes an increase of the number of men affected by benign prostatic hyperplasia $(\mathrm{BPH})$ with a large number of pathology associated that may limit its surgical treatment. In this study we try to show the effectiveness and security of local anaesthesia and sedation in the endoscopic treatment of the BPH in patients with high surgical-anaesthetic risk.

Methods: During the last 4 years, 38 patients underwent surgical treatment of BPH with ages between 78 and 86 years (average $81,4 \pm 3,2$ ) with severe obstructive prostatic syndrome and high surgical-anaesthetic risk. 15 patients underwent, transurethral incision of prostate (TUIP) and in 23 patients, transurethral resection (TURP) was performed. The blockade anesthetic was made by transrectal periprostatic infiltration finger-guided with 10-15 millilitres of lidocaine $2 \%$. During the procedure an intravenous perfusion of propofol and remifentanil was administered. The obtained results as well as the patient's satisfaction degree was evaluated by means of the elaboration of a questionnaire .

Results: The average length of the surgical treatment was of $12 \pm 5$ minutes (rank $8-25$ ), having itself not stated complications derived from the anesthetic technique. They referred a slight annoyance 4 patients (11\%) that was treated increasing the rate of the sedative perfusion. The satisfaction's degree with the received treatment has been good in the $95 \%$ of patients.

Conclusions: We considered that the TUIP as much as TURP made under local anesthesia with sedation are safe, effective methods and well tolerated, that fundamentally are indicated in aged patients and when the surgical-anesthetic risk is elevated.

Key words: Benign prostatic hyperplasia. Local anaesthesia. Transurethral prostatic resection. 
$\mathrm{E}$ aumento progresivo de la esperanza de vida está contribuyendo a que un número paulatinamente creciente de pacientes con sintomas de obstrucción del tracto urinario inferior y múltiple patología asociada acuda a las consultas de urología. Por otra parte, aunque en la última década el mercado tecnológico haya desarrollado, más bien con poca fortuna, diversos métodos alternativos a la resección transuretral prostática (RTUP), ésta continúa siendo el procedimiento de elección para el tratamiento quirúrgico de la hiperplasia benigna de próstata (HBP), constituyendo asimismo el "patrón de oro" con el que se comparan todas las demás técnicas. No obstante, su empleo se encuentra limitado en aquellos pacientes que presentan un elevado riesgo para la utilización de las técnicas convencionales de anestesia (regional o general). En este contexto, se han publicado diversos estudios que han puesto de manifiesto la eficacia y baja morbilidad tanto de la incisión transuretral prostática (ITUP) como de la RTUP realizadas bajo anestesia local y control anestésico monitorizado, en los que la asociación de infiltración anestésica local de la próstata (para la que se han descrito diferentes procedimientos) junto con la administración intravenosa de una perfusión sedante, parece proporcionar un buen control del dolor intraoperatorio, así como un excelente grado de satisfacción de los pacientes sin afectar a los resultados de la técnica quirúrgica ni aumentar el índice de complicaciones $^{1-3}$.

\section{MATERIAL Y MÉTODOS}

Durante los últimos 4 años hemos intervenido quirúrgicamente en nuestro centro a 38 pacientes afectos de sindrome de prostatismo obstructivo severo (16 con sondaje vesical permanente y 22 con una puntuación superior a 20 del índice internacional de sintomas prostáticos IPSS) bajo anestesia local asistida, con edades comprendidas entre 78 y 86 años (media 81,4 $\pm 3,2$ ). El volumen medio prostático, evaluado mediante ecografía transrectal preoperatoria, osciló entre 25 y 58 g. (media 36,2 $\pm 6,1$ ).

En cuanto al procedimiento quirúrgico empleado, en 15 (39\%) pacientes se practicó ITUP con asa de Collins y en los 23 (61\%) restantes se efectuó RTUP con asa tipo Wing.
La valoración del riesgo quirúrgico-anestésico de los pacientes se realizó según la clasificación de la Asociación Americana de Anestesiología, siendo 26 pacientes los catalogados como ASA III (68\%) y 12 ASA IV (32\%).

En cuanto a la técnica anestésica utilizada, en un primer momento se procede a la instilación intrauretral de $5 \mathrm{ml}$ de gel de lidocaína al 2\%, siendo retenidos en la uretra mediante la colocación de una pinza peneana durante unos 20-30 minutos previos a la intervención. Para el bloqueo anestésico prostático realizamos la infiltración de unos 10-15 cc. de lidocaína al 2\% por vía transrectal y digito-dirigida con una aguja de anestesia intradural de calibre 20G. Las inyecciones las practicamos a ambos lados del ápex prostático (aproximadamente a las 5 y 7 horarias) y en la base de la próstata de forma bilateral, a nivel de la localización teórica de las bandeletas neurovasculares (Fig. 1). Tras la inserción del resector, inmediatamente antes de comenzar la intervención, se procede a la administración intravenosa de una perfusión continua de propofol y remifentanilo, fármacos con efecto hipnótico y analgésico respectivamente. Durante todo el procedimiento, el anestesista mantiene un control monitorizado de las constantes del paciente.

Para controlar el dolor durante el postoperatorio, en todos los casos se administraron $2 \mathrm{~g}$ de metamizol intravenoso cada 6 horas durante las primeras 24 horas del postoperatorio, utilizando como analgésico de rescate 1-2 cc de meperidina subcutánea. En cuanto a la profilaxis antibiótica se administraron $2 \mathrm{~g}$ de cefotaxima o $240 \mathrm{mg}$ de gentamicina endovenosos.

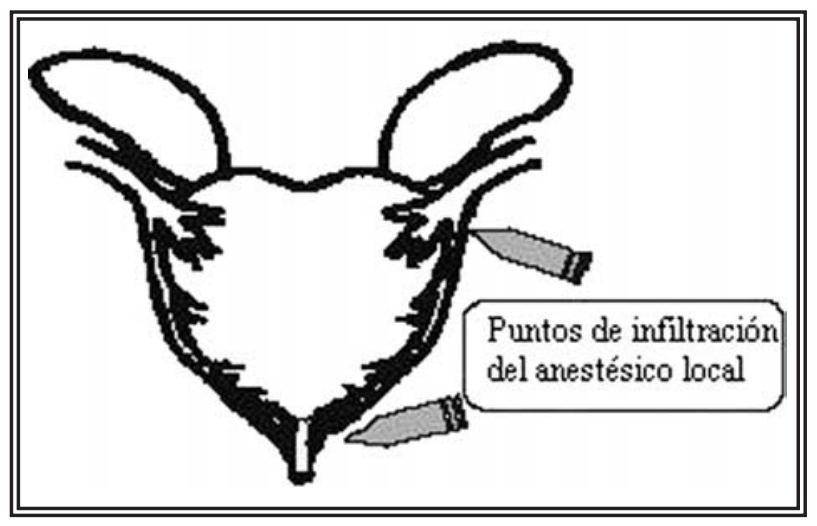

FIGURA 1. Representación esquemática de los puntos de infiltración anestésica. 
En el momento del alta hospitalaria, se realizó un cuestionario para evaluar la intensidad del dolor, tanto durante el procedimiento quirúrgico como en el postoperatorio, con una escala de 0 10 puntos $(0=$ no dolor, $1-2=$ molestia leve, $3-5$ = dolor leve, $6-8=$ dolor moderado, $9-10=$ dolor severo). Asimismo, en la revisión realizada al mes de la intervención se valoró el grado de satisfacción con el tratamiento recibido expresado por los pacientes $(1=$ deficiente, 2 = regular, $3=$ bueno, 4 = excelente).

\section{RESULTADOS}

En todos los casos hubo una buena tolerancia al procedimiento no precisándose en ninguno de ellos la reconversión del tipo de anestesia. La mayoría de los pacientes, 34 (89\%), no presentaron dolor durante la intervención; sin embargo, los 4 restantes (11\%) refirieron una molestia leve, que se controló con éxito aumentando la velocidad de perfusión del compuesto sedante.

Durante el postoperatorio, el dolor fue controlado eficazmente con analgésicos menores en 30 pacientes (79\%); no obstante, los 8 restantes (21\%) presentaron dolor moderado o severo que obligó a la administración de 1-2 cc de meperidina subcutánea durante las primeras 12 horas tras la intervención.

La duración del acto quirúrgico osciló entre 8 y 25 minutos (media $12 \pm 5$ minutos), siendo el volumen medio resecado de $24 \pm 6 \mathrm{~g}$, en aquellos casos en los que se realizó RTUP.

No evidenciamos en ningún caso complicaciones derivadas de la técnica anestésica ni de la sedación. La tolerancia líquida se inició a las 2 horas de la intervención. La hematuria cedió generalmente en las primeras 24 horas, persistiendo en 6 pacientes (16\%) hasta el segundo día del postoperatorio, no precisándose la transfusión sanguínea en ninguno de ellos. El catéter vesical se retiró cuando cedió la hematuria, a las 24 horas en 32 pacientes (84\%) y en los 6 restantes (16\%) a las 48 horas. La estancia postoperatoria fue de 2 días en todos los casos.

En la revisión realizada al mes de la intervención, la totalidad de los pacientes presentaban una evolución satisfactoria, con micción espontánea en todos los casos, así como una mejoría tanto subjetiva como objetiva del flujo urinario, siendo el pico de flujo máximo (Qmax) medio de $15 \pm 3 \mathrm{ml} / \mathrm{seg}$; contestando 36 de ellos (95\%) la encuesta sobre el grado de satisfacción con el tratamiento recibido, cuyos resultados se detallan en la Tabla 1.

Tabla 1

Satisfacción con el tratamiento recibido

\begin{tabular}{lcc}
\hline Calificación & No casos & \% \\
\hline Excelente & 28 & $78 \%$ \\
Buena & 6 & $17 \%$ \\
Regular & 2 & $5 \%$ \\
Deficiente & 0 & $0 \%$ \\
\hline
\end{tabular}

\section{DISCUSION}

El actual envejecimiento de la población en nuestro entorno constituye la causa fundamental del progresivo incremento de pacientes afectos de HBP, en los que el tratamiento médico resulta insuficiente para el control de los síntomas y son candidatos a ser desobstruidos quirúrgicamente. No obstante, cualquier actuación terapéutica agresiva en estos varones de edad avanzada suele representar una elevada morbilidad como consecuencia de la frecuente asociación de múltiple patología concomitante, que desaconseja o incluso contraindica la realización de los procedimientos anestésicos regionales o generales habituales, lo que limita nuestro arsenal terapéutico y produce un impacto considerable sobre la calidad de vida de estos pacientes. Por otra parte, el desarrollo de nuevos procedimientos en el contexto de la anestesia local junto con la aparición de nuevos fármacos sedantes más efectivos y seguros, han demostrado la posibilidad de realizar un bloqueo prostático eficaz y un efecto analgésico suficiente, sin exponer al paciente a los riesgos de la anestesia convencional.

Las diversas técnicas que se han descrito para el bloqueo prostático se fundamentan en la infiltración de anestésicos locales a nivel del plexo pélvico, el cual forma parte de las denominadas bandeletas neurovasculares. Estas bandeletas se localizan de forma bilateral a la próstata, la cual recorren en posición posterolateral descansando sobre la fascia endopélvica lateral y la de Denonvillers hasta alcanzar el ápex prostático, donde se sitúan en la posición 5 y 7 horarias $^{1}$ (Fig. 2). 


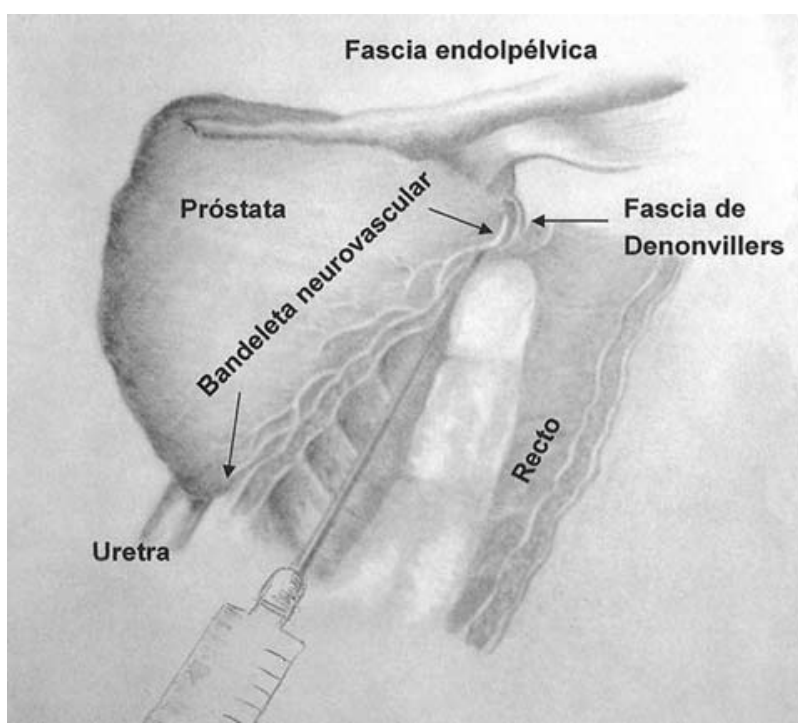

FIGURA 2. Esquema de la técnica de infiltración periprostática digito-dirigida.

Como anestésico local preferimos la lidocaína a los otros compuestos amídicos comúnmente empleados (mepivacaína, bupivacaína y ropivacaína), pues tiene menor efecto cardiotóxico en caso de entrada directa del fármaco al torrente vascular, tiene una acción anestésica más rápida y es la que provoca una sensación menos dolorosa durante su infiltración ${ }^{2}$. También preferimos su utilización por tratarse del mismo compuesto que posee el gel anestésico que previamente hemos instilado a través de la uretra del paciente para anestesiar la mucosa uretrovesical. Tiene el inconveniente de provocar un tiempo de acción anestésica mas breve que el resto, pero habitualmente se trata de intervenciones de corta duración que en nuestra experiencia ha sido siempre inferior a media hora y en ningún caso hemos tenido que repetir la infiltración por agotarse el efecto anestésico antes de finalizar la intervención.

En algunas publicaciones se recomienda la asociación de un vasoconstrictor al anestésico local infiltrado con el fin de disminuir el riesgo de hemorragias $^{3,4}$. No obstante, en nuestra experiencia, que prescindimos de dicha combinación, no parece haberse traducido en un aumento del sangrado intraoperatorio que interfiriera con el procedimiento. Tampoco parece que hayamos tenido un aumento significativo del número de pacientes con hematuria ni de la intensidad de la misma en comparación con otras series, por lo que creemos que la utilización de vasoconstrictores puede ser obviada al no aportar un claro beneficio adicional y exponer a riesgos innecesarios al paciente.

Algunos autores han propugnado la infiltración del cuello vesical asociada al bloqueo prostático para la realización de ITUP y de RTUP sin que aparentemente su utilización consiga un control del dolor más eficaz, incluso se ha relacionado su uso con la aparición de retención aguda urinaria postoperatoria ${ }^{5}$, razón por la que, como la mayoría de autores revisados, no sólo la creemos innecesaria sino desaconsejable ${ }^{3,6,7}$.

La práctica de RTUP con anestesia local fue descrita inicialmente por Moffat en 1977 mediante la infiltración de lidocaína al $1 \%$ por vía transperineal e instilación intrauretral de un anestésico tópico, asociando sedantes intravenosos en los pacientes que presentaban dolor ${ }^{8}$. Desde entonces, diversas publicaciones han sugerido la utilización de este procedimiento con buenos resultados para el control del dolor y con baja morbilidad independientemente tanto del anestésico utilizado como de la vía de acceso para el bloqueo del plexo pélvico ${ }^{3,6-9}$. Nosotros preferimos el acceso transrectal digito-dirigido, ya que en nuestra opinión es un método más rápido, menos doloroso y permite localizar con mayor exactitud las zonas a anestesiar que con otras técnicas.

En lo que se refiere al tamaño prostático, es aconsejable la selección de glándulas pequeñas o medianas que no superen los 60 gramos, ya que obviamente, un mayor volumen prostático se corresponde con un tiempo de resección superior, y por lo tanto, más probabilidades de complicaciones, sobre todo si tenemos en consideración que se trata de pacientes de alto riesgo y con elevada morbilidad añadida. No obstante, algunos autores han publicado resecciones de próstatas de gran volumen realizando un drenaje suprapúbico peroperatorio (habitualmente con el trocar de Reuter) que evita la distensión vesical haciendo la resección más sencilla y aumentando la seguridad operatoria ${ }^{10}$. Siguiendo con este criterio, el grupo del Hospital Clínico de Zaragoza ha informado de resecciones de próstatas de hasta 250 gramos utilizando un tubo de Amplatz suprapúbico $^{11}$, cuyo gran calibre, permite mante- 
ner una presión de irrigación inferior a la presión venosa, previniendo el síndrome de reabsorción a la vez que permite la aspiración de los fragmentos resecados, ahorrando tiempo y evitando las hemorragias que se producen habitualmente durante la manipulación de la irrigación transuretral.

Por lo general, preferimos la RTUP a la ITUP, pero en casos de próstatas de pequeño volumen y cuando el riesgo quirúrgico es extremadamente elevado, habitualmente optamos por realizar esta última en base a que precisa un menor tiempo operatorio así como un menor riesgo de hemorragia postquirúrgica, sobre todo si la incisión se realiza de forma unilateral ${ }^{12}$.

Respecto a la ITUP, algunos autores han propugnado su realización mediante la aplicación de anestesia local tópica asociada a infiltración intrauretral a nivel del trayecto a escindir ${ }^{4,5}$; sin embargo, nosotros consideramos más apropiada la práctica de un bloqueo prostático bajo control anestésico monitorizado en base a los resultados obtenidos por estos autores, en los que un elevado número de pacientes (17\%) refirió un dolor severo durante el acto quirúrgico y hubieran preferido una anestesia general, aún cuando habían recibido una dosis preoperatoria de opiáceos y benzodiacepinas. Por otra parte, se han publicado diferentes trabajos en los que la ITUP se ha mostrado superior a la RTUP en cuanto a la mejoría subjetiva de la sintomatología y parece ser tan eficaz como ésta con seguimientos de hasta 2 años respecto al aumento del pico de flujo ${ }^{13-15}$; sin embargo, no se aconseja su práctica en pacientes con sintomatología fundamentalmente irritativa, en los que la RTUP se ha mostrado más eficaz, probablemente por eliminar focos de prostatitis y conseguir un mayor grado de desobstrucción ${ }^{5,16}$. Su principal inconveniente es la incapacidad para realizar un estudio histológico prostático, lo que puede dejar sin diagnosticar un proceso neoplásico subyacente. No obstante, su realización la creemos fundamentalmente indicada en pacientes con un riesgo quirúrgico muy elevado y en pacientes con próstatas pequeñas, ya sea con incisión uni o bilateral, asî como asociada a la RTUP en glándulas de mayor tamaño en las que no sea conveniente alargar el tiempo operatorio.
Para la práctica de la RTUP preferimos el uso de asas tipo Wing a las asas convencionales, ya que con las primeras, al realizar una función doble de resección y vaporización, proporcionan un mayor rendimiento operatorio, resecando mayor cantidad de tejido en menor tiempo a la vez que realizan mejor hemostasia. Asimismo, al estar los fragmentos prostáticos parcialmente vaporizados, estos son de menor tamaño y por tanto de más fácil extracción por lavado ${ }^{17}$. En este contexto, recientes publicaciones parecen poner de manifiesto que la utilización de generadores bipolares por pulsos mejoran sustancialmente el rendimiento de la resección sin mermar en absoluto ninguna de las magníficas cualidades de la RTUP; asimismo, el hecho de poder utilizar solución salina como líquido de irrigación, minimiza el riesgo de aparición de síndrome de reabsorción ${ }^{18-20}$.

En cuanto al tiempo de permanencia postoperatoria de la sonda, aunque algunos autores han propuesto la posibilidad de retirarla de forma precoz durante las primeras horas tras la intervención, sin aumentar de forma considerable el riesgo de retención urinaria ${ }^{13}$, nosotros preferimos mantenerla durante al menos 24 horas siempre que no exista hematuria, con el objetivo de disminuir en la medida de lo posible la retención urinaria, ya que en nuestra opinión, esta complicación afecta en gran medida la confianza en el procedimiento y el grado de satisfacción del paciente con el tratamiento recibido.

Podemos concluir afirmando que tanto la ITUP como la RTUP realizadas bajo anestesia local y sedación son métodos seguros, eficaces y con buena aceptación en pacientes seleccionados susceptibles de ser intervenidos por obstrucción del cuello vesical. En nuestra opinión existe una clara indicación de este procedimiento en pacientes añosos y en aquellos en los que exista un riesgo quirúrgico - anestésico elevado (ASA III-IV), donde las técnicas anestésicas tradicionales suelen estar contraindicadas; hecho por el cuál, ésta técnica debería formar parte de la cartera de procedimientos habituales del urólogo.

\section{REFERENCIAS}

1. Oliveira A, Tobias M, Roger E. Prostate innervation and local anesthesia in prostate procedures. Rev Hosp Clín Fac Med. S Paulo 2002;57:287-292. 
2. De Andres J, Valía JC. Anestésicos locales. En: Torres Morera LM. Tratado de anestesia y reanimación; Ed. Aran SA; Madrid 2001;967-982.

3. Chander J, Gupta U, Mehra R, Ramteke VK. Safety and efficacy of transurethral resection of the prostate under sedoanalgesia. BJU Int 2000;86:220-222.

4. Irani I, Bon D, Fournier F, Dore B, Aubert J. Patient acceptability of transurethral incision of the prostate under local anaesthesia. Br J Urol 1996;78:904-906.

5. Hugosson J, Bergdahl S, Norlen L. Outpatient transurethral incision of the prostate under local anesthesia: operative results, patient security and cost effectiveness. Scand J Urol Nephrol 1993;27:381-385.

6. Birch BR, Gelister JS, Parker CJ, Chave H, Miller R. TURP under sedation and local anesthesia (sedoanalgesia). Experience in 100 patients. Urology 1991;38:113-118.

7. Akalin Z, Mungan NA, Basar H, Aydioganli L, Cengiz T. Transurethral resection of the prostate and laser prostatectomy under local anesthesia. Eur Urol 1998;33:202-205.

8. Moffat NA. Transurethral prostatic resection under local anesthesia. J Urol 1977;118:607-608.

9. Tabet BG, Levine S. Nerve block in prostate surgery. J Urol 1996;156:1659-1661.

10. Reuter HJ, Jones LW. Physiologic low presure irrigation for transutretrhal resection: suprapubic trocar drainage. J Urol 1974;111:210-212.

11. Sánchez JM, Sánchez MA, Regojo O, Elizalde B, Valle J, López JA, et al. Técnica modificada para la resección transuretral de próstata con drenaje suprapúbico y anestesia local. Actas Urol Esp 2003;27:216-220.

12. Blasco JE, Gonzalvo V, Navalón P. Trigonocervicoprostatotomía endoscópica: Nuestra experiencia. Actas Urol Esp 1993; 17:315-318.
13. Chander J, Vanitha V, Lal P, Ramteke VK. Transurethral resection of the prostate as catheter-free day-care surgery. BJU Int 2003 Sep;92:422-425.

14. Katz PG, Greenstein A, Ratliff JE, Marks S, Guice J. Transurethral incision of the bladder neck and prostate. J Urol 1990;144:694-696.

15. Orandi A. Transurethral incisión of prostate compared with transurethral resection of the prostate in 132 matching cases. J Urol 1987;138:810-815.

16. Dörflinger T, Ester M, Larsen JF. Transurethral prostatectomy or incision of the prostate in the treatment of prostatism caused by small benign prostate. Scand J Urol Nephrol 1987;104:77-81.

17. Ramos M, Tramoyeres A, Navalón P. La electrovaporización con asa Wing frente a la resección convencional: estudio de eficacia y seguridad. Actas Urol Esp 2001;25(6 supl):144.

18. Valdivia JG, Regojo O, Sánchez JM, Elizalde A, Navarro J, Hijazo I. La resección trasuretral con solución salina: un logro tecnológico aún no asimilado por la urología. Arch Esp Urol 2005;58:335-345.

19. Botto H, Lebret T, Marré PH. Electrovaporization of the prostate with the Gyrus device. J Endourol 2001;15:313320.

20. Eaton AC, Francis RN. The provision of transurethral prostatectomy on a day-case basis using bipolar plasma kinetic technology. BJU Int 2002;89:534-541.

Dr. P. Navalón Verdejo

Email: pedronavalon@yahoo.com

(Trabajo recibido el 7 de septiembre 2004)

\section{COMENTARIO EDITORIAL}

No cabe duda que resolver la obstrucción infravesical, por HBP en pacientes de edad avanzada y alto riesgo anestésico es un reto que tenemos que encarar cada vez con mayor frecuencia. En este trabajo los autores solventan este problema de forma eficaz en 38 pacientes, con edad media superior a los 80 años y ASA III-IV, mediante RTU (23 casos) e ITU (15 casos) bajo anestesia local y sedación. El tiempo operatorio empleado fue pequeño $(<15 \mathrm{~min})$ y ningún paciente sufrió complicaciones. Hay, pues, que felicitarles por ello.

Ahora bien, habria que hacer alguna observación al respecto. La RTU de próstata es una intervención que no está exenta de complicaciones intra y perioperatorias, y algunas de ellas no dependen del tipo de anestesia empleada. La bacteriemia o el sangrado, son frecuentes durante la RTU y parecen disminuir con la enucleación con láser. Llama la atención en esta serie de pacientes, el escaso sangrado (el $84 \%$ de los pacientes tuvieron orinas hematúricas sólo un día), y la ausencia de sintomas irritativos tras la retirada precoz de la sonda uretral, que en la mayoría de los pacientes se hizo a las $24 \mathrm{~h}$ de la intervención.

Fdo.: Dr. J.H. Amón Sesmero

\section{CONTESTACION AL COMENTARIO EDITORIAL}

Agradecemos las felicitaciones del Comité Editorial, aunque consideramos que resolver con éxito 38 casos de obstrucción infravesical en pacientes muy seleccionados, con próstatas de pequeño volumen, no representa ningún hecho extraordinario.

No cabe duda que en nuestra experiencia también hemos tenido complicaciones inherentes a 
la RTUP, fundamentalmente hematurias anemizantes postoperatorias que han precisado transfusión, así como, alteraciones secundarias a la reabsorción del líquido de irrigación, aunque estas complicaciones habitualmente se asocian con resecciones prolongadas de grandes adenomas, pero en los casos que presentamos en este estudio, procuramos tomar todas las medidas disponibles para minimizar riesgos, realizando ITUP sólo en un lado para que el lecho cruento sea lo más pequeño posible y reducir asimismo su tiempo de ejecución, ya que dicha incisión puede ser realizada con seguridad en muy pocos minutos por cualquier urólogo medianamente experimentado. Igualmente, en algunas ocasiones, cuando la próstata es de mayor tamaño, realizamos RTUP de un lado e ITUP del otro; y en caso de próstatas trilobuladas, propugnamos la resección del lóbulo medio y la incisión de uno de los lóbulos laterales, con lo que obviamente reducimos de forma considerable la probabilidad de complicaciones.

Nosotros también creemos que algunos de nuestros pacientes se hubieran podido benefi- ciar con la tecnología láser, pero como es obvio, para la utilización de cualquier técnica se precisa de los dos indicadores de estructura convencionalmente usados: disponibilidad y accesibilidad. En el momento que realizamos el estudio, nuestra disponibilidad era nula, ya que no poseíamos dicha tecnología en nuestra comunidad. Desconocemos la accesibilidad (económica) por parte de los pacientes. No obstante, en la actualidad, a la hora de redactar el presente escrito, ya tenemos experiencia con esta tecnología, y aunque nuestra casuística es todavía muy escasa como para inferir conclusiones definitivas, parece que no es una técnica tan sencilla e inocua como se ha publicitado; asimismo, si además tenemos en consideración factores tales como coste-efectividad de los procedimientos o los índices de utilización de quirófanos (PIQ), estamos seguros que la RTUP resultaría favorecida al comparar ambas técnicas.

Fdo.: Dr. P. Navalón Verdejo 